\title{
Synthesis of Enantioenriched Tetra-ortho-3,3'-Substituted Biaryls by Small-Molecule-Catalyzed Noncanonical Polyketide Cyclizations
}

\author{
Reto M. Witzig, \\ Christof Sparr* \\ Department of Chemistry, University of Basel, \\ St. Johanns-Ring 19, 4056 Basel, Switzerland \\ christof.sparr@unibas.ch
}

Abstract The arene-forming aldol condensation is a fundamental reaction in the biosynthesis of aromatic polyketides. Strictly controlled by the polyketide synthases, the highly reactive poly- $\beta$-carbonyl substrates are diverged into numerous aromatic natural products by selective cyclization reactions; a fascinating biosynthetic strategy that sparked our interest to investigate atroposelective aldol condensations. In this account, we contextualize and highlight the ability of small-molecule catalysts to selectively convert noncanonical hexa-carbonyl substrates in a double arene-forming aldol condensation resulting in the atroposelective synthesis of tetra-ortho-3,3'substituted biaryls. The hexa-carbonyl substrates were obtained by a four-fold ozonolysis enabling a late-stage introduction of all carbonyl functions in one step. Secondary amine catalysts capable of forming an extended hydrogenbonding network triggered the noncanonical polyketide cyclization in order to form valuable biaryl in high yields and with stereocontrol of up to 98:2 e.r.

Key words aldol condensation, atropisomerism, ligand design, polyketides, stereoselective catalysis

\section{Biosynthesis of Aromatic Polyketides}

The exceptional reaction control of enzymes can be well exemplified by the biosynthesis of aromatic polyketides. Precise modifications and selective cyclization reactions of polycarbonyl chains induced by an intricate enzymatic machinery allow the diversification of common precursor molecules to one of the largest families of natural products. The polyketide biosynthesis can be divided into three stages (chain assembly, cyclization and tailoring steps), whereas divergence is achieved in every phase.[13] Polyketide natural products are produced by polyketide synthases (PKSs), multifunctional enzymes which have been classified into different families.[4,5] Type I PKSs contain covalently linked multidomain enzymes while type II and III generally consist of dissociable monofunctional enzymes. The polyketide synthases belonging to type II and III operate in an iterative fashion, thus multiple transformations are repeated at the active sites. On the other hand, in the non-iterative type I PKSs, every enzyme domain is catalyzing a specific

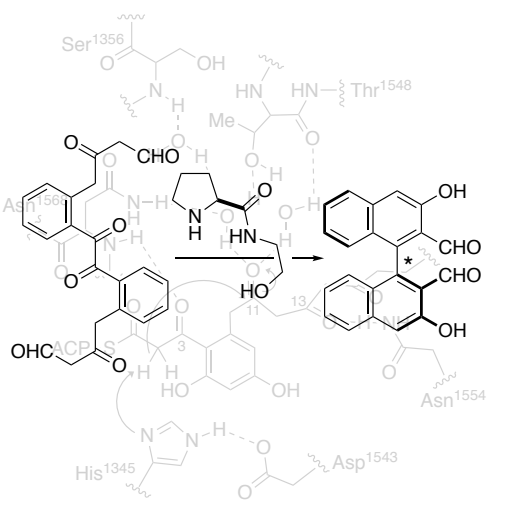

transformation. In a first assembly phase, repetitive decarboxylative Claisen type reactions of activated acetate units (for example malonyl $\mathrm{CoA}$ ) allow the preparation of poly- $\beta$ carbonyl substrates. For instance, non-reduced poly- $\beta$-carbonyl chains are obtained by minimal PKSs consisting of two ketosynthases (KS) and an acetyl carrier protein (ACP). After the first diversification obtained through variations in the starting units (Figure 1, starting unit $=\mathrm{R}$ ), a second differentiation is gained by the chain length which is controlled by the two KS in the minimal PKS. Further variation is attained by partial or full reduction of the appended carbonyl function which are optionally obtained through additional ketoreductases (KR), dehydratases (DH) and enoyl reductases (ER). Depending on the degree of reduction, the domains are classified as non-reducing (NR), partially- (PR), highly- (HR) or fully-reducing (FR).[3]

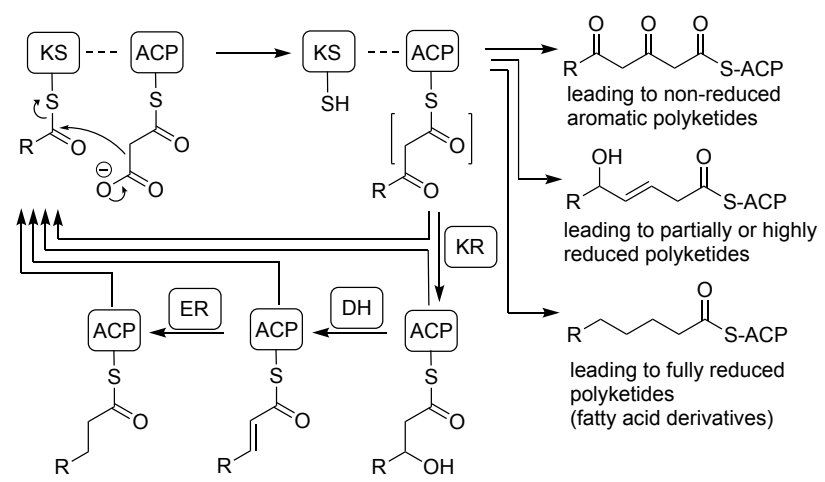

Figure 1: Simplified overview of the diversification of the poly- $\beta$-carbonyl substrates based on different starting units, variation of the chain length and partial to full reduction of the appended carbonyl functions.

While a full reduction of the extended carbonyl function leads to a two-carbon extension of the saturated fatty acid chain, the assembly of non-reduced poly- $\beta$-carbonyl chain results in highly 
reactive compounds which can undergo a variety of different cyclizations. Stabilization of the acyclic intermediates is essential in order to prevent spontaneous cyclizations and to maintain the control over the diversification. The ensuing cyclization of poly$\beta$-carbonyl chains offers an enormous potential for structural variation, which becomes evident by the conversion of an unreduced tetraketide precursor by chalcone synthases. Three different cyclization modes can be obtained from this short poly$\beta$-carbonyl substrate. An aldol-type cyclization is leading to the formation of a stilbene, while the chalcone structure is resulting from a Claisen-type cyclization. A third cyclization possibility is the pyrone formation (Figure 2).[6] Conceivably, longer substrate chains significantly increase the number of possible cyclizations and therefore increase the need to keep the cyclizations under strict control.

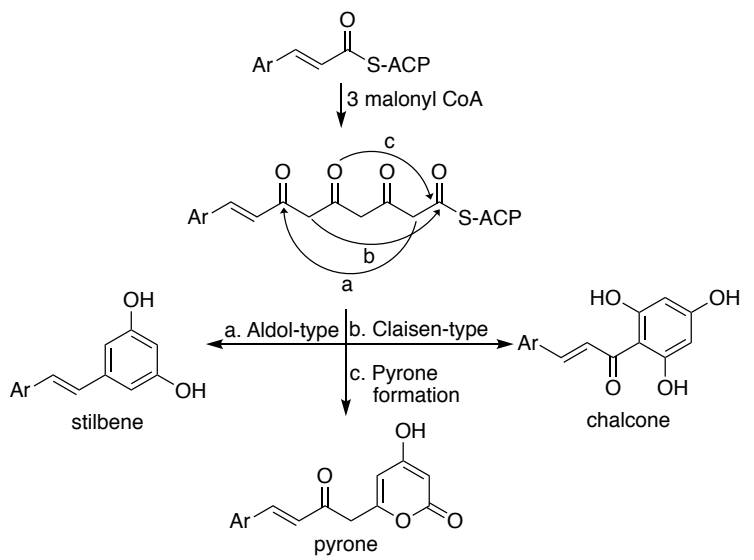

Figure 2: Three possible cyclizations of an unreduced tetraketide substrate.

Compared to the well explored assembly of the poly- $\beta$-carbonyl chain substrates, the detailed operation modes of enzymes to stabilize and selectively cyclize these highly reactive intermediates is less established. The Townsend group investigated the cyclization of an octaketide to an anthrone intermediate in the biosynthesis of Aflatoxin $\mathrm{B}_{1} .^{[7]}$ Thereby, they deconstructed an iterative multidomain of the polyketide synthase and investigated the individual roles of each domain unit. A product template domain (PT) was discovered, which

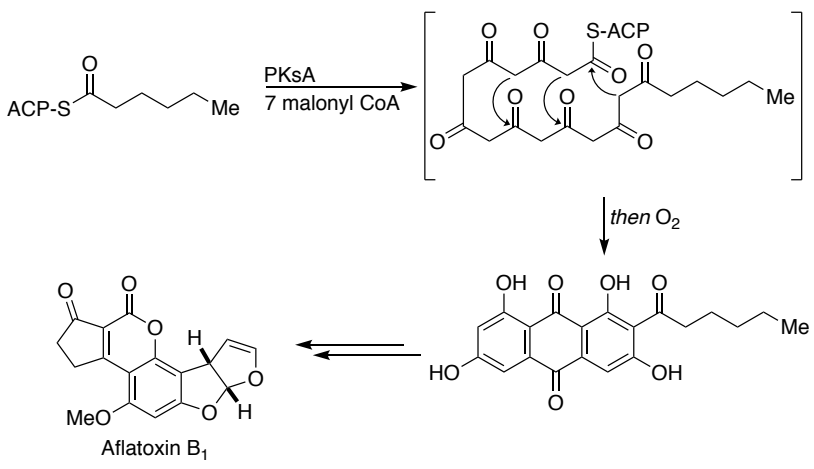

Figure 3: Assembly of seven malonyl CoA units and three-fold cyclization followed by an autooxidation to norsolorinic acid in the biosynthesis of Aflatoxin B1.

together with the KS and a thioesterase, significantly enhanced the assembly of seven malonyl CoA units and mediated specific cyclizations to the first three rings (Figure 3). The apparent importance of the PT encouraged them to pursue a further exploration of this enzyme domain. The researchers were able to co-crystallize the PT domain with palmitate as an analog for the carbonyl chain substrate and a naphthalene compound as product mimic. ${ }^{[8]}$ The active site of the domain was identified from analysis of the X-ray structure as both co-crystals were located in the same region of the domain. Due to the minimal conformational change between the two crystal structures, which virtually contain the substrate and product of the polyketide cyclization in the active site, they proposed the occurrence of the cyclizations within the detected enzyme pocket. The active site consists of a hydrophilic site to stabilize the carbonyl functions and an opposite hydrophobic site to coordinate the cyclization products. The cyclization chamber in the middle of the pocket suggests a pre-organization of the substrate chain through a "kink", thereby arranging the C4 in close proximity of the proposed catalytic dyad with His ${ }^{1345}$ and Asp ${ }^{1543}$ to undergo a first arene-forming aldol condensation with the ketone C9 (Figure 4a). The obtained arene is proposed to be further transformed in a similar aldol condensation between the C2 and C11 (Figure 4b).
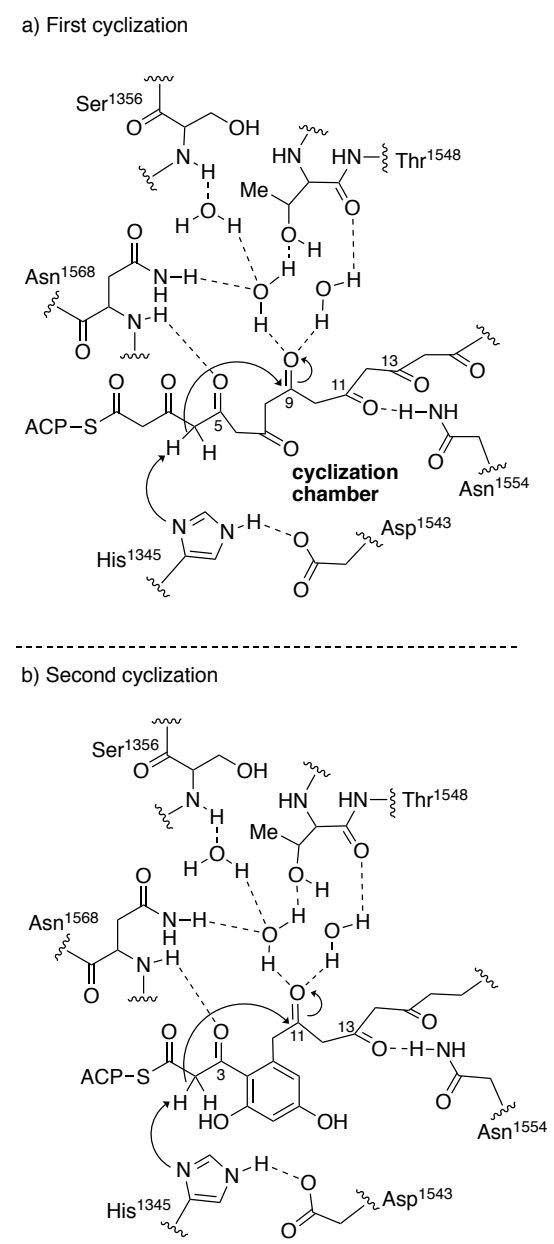

Figure 4: Proposed mechanism for the selective cyclization in the PT domain. The stereogenic centers are omitted for clarity. 
Based on the similar domain organization of other non-reducing PKS, the proposed mechanism was hypothesized as being general for non-reducing PKS. The highly optimized enzyme pocket which folds the poly- $\beta$-carbonyl substrate for controlled cyclizations indicates the challenge to handle these highly reactive intermediates.

\section{Rotationally Restricted Aromatic Polyketides}

Post assembly modifications further extend the diversity of aromatic polyketide natural products. The so-called tailoring steps typically involve glycosylation reactions and various oxidations.[9,10] Particularly interesting are oxidative dimerizations rendering the possibility to form rotationally restricted biaryl compounds in diastereoselective[11] or sometimes even enantioselective processes.[12] These radical mediated reactions are catalyzed by oxidative enzymes such as laccases, peroxidases or cytochrome $\mathrm{P} 450$ and give rise to a vast variety of dimers due to the stabilization and reactivity of the radical intermediates at the oxygen atom as well as the ortho- and para-position of the phenol (Figure 5).[13]

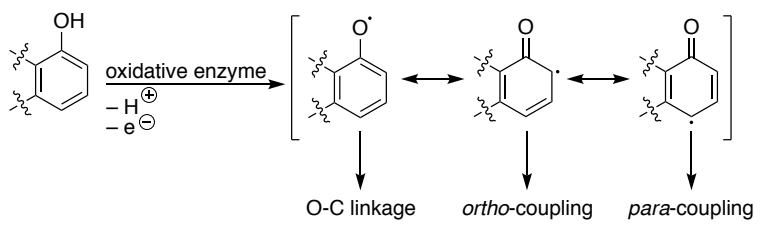

Figure 5: Stabilized radicals resulting in O-C linkage, ortho- or paraphenol couplings.

The Müller group explored the responsible gene clusters for the enantioselective dimerization of 7-demethylsiderin and detected a cytochrome gene KtnC being essential for the symmetric orthophenol coupling to $\left(S_{\mathrm{a}}\right)$-Orlandin, which is an intermediate in the biosynthesis of Kotanin. ${ }^{[14]}$ More recently, they could express the enzymes of $\mathrm{KtnC}$ in order to demonstrate that the enantioselectivity observed in the reaction is independent from other enzymes. Furthermore, the in vivo expression of the enzyme homologue DesC catalyzed the dimerization to $\left(R_{\mathrm{a}}\right)$ Desertorin A which is a regioisomer of $\left(S_{\mathrm{a}}\right)$-Orlandin, exhibiting the divergence in the biosynthesis of aromatic polyketides in tailoring processes (Figure 6).[15]

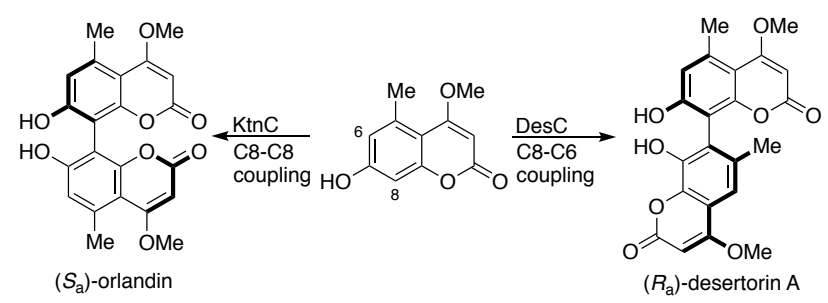

Figure 6: The oxidative dimerization of 7-demethylsiderin to (Sa)Orlandin catalyzed by $\mathrm{KtnC}$ or to (Ra)-Desertorin using the enzyme homologue DesC.

Until recently, rotationally restricted aromatic polyketides were assumed to be exclusively formed through oxidative coupling processes. However, compared to the regular 1,4 and 1,6 phenol connectivity resulting from oxidative dimerization, the rather unusual pentacyclic fasamycins and related members possess a 1,5-oxygenation pattern which suggests an alternative mechanism for the formation of the rotationally restricted axis (Figure 7).[16]

a) Oxygenation pattern from dimerizations<smiles>Cc1cc(O)cc(O)c1-c1c(O)cc(O)c(I)c1I</smiles><smiles>COc1cc(Cc2cc(O)cc3cc4c(cc23)C(=O)c2c(O)cc(O)cc2C4(C)C)cc(OC)c1</smiles>

Figure 7: a) Oxidative dimerization resulting in a 1,4 and 1,6 phenol connectivity; b) A 1,5 phenol connectivity in Fasamycin C.

Brady and co-workers proposed that the biosynthetic pathway of related polyketide natural products proceeds through the assembly of a non-reduced trideca-ketide by a minimal PKS. Three initial cyclizations (C5 to C18, C7 to C16 and C9 to C14) were proposed being similar to the biosynthesis of $\gamma$ Rubromycin, Fredericamycin, Griseorhodin, Benestatin based on the closely related KS genes.[17] Intriguingly, the configuration of the stereogenic axis is defined in the two enzyme-catalyzed cyclizations (C2 to C19 and C20 to C25). Thus, the PKS not only catalyzes a regioselective but even an atroposelective areneforming aldol condensation. Further tailoring steps such as a decarboxylation, methylation and halogenation finally yield Fasamycin B (Figure 8).[16]
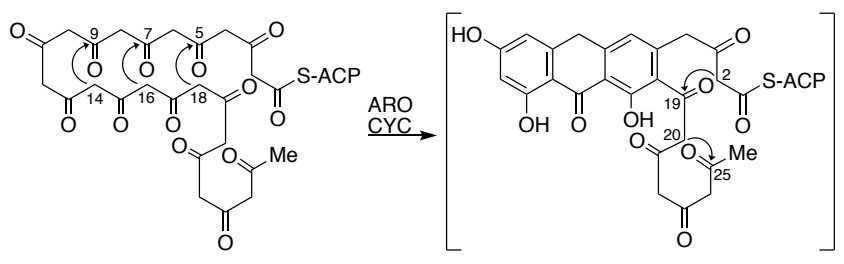

ARO/CYC
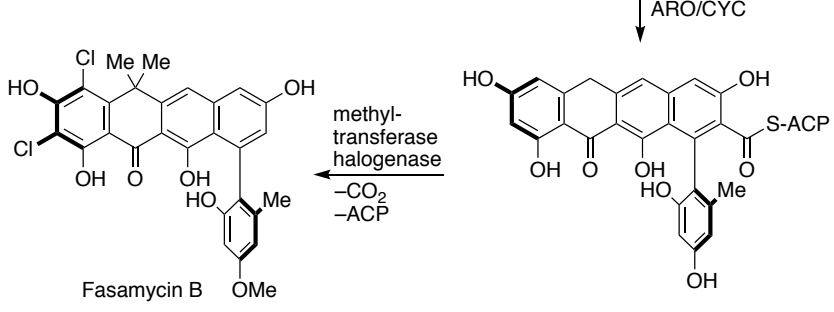

Figure 8: Proposed biosynthesis of Fasamycin A by Brady and coworkers. ${ }^{[20-21]}$

Beyond the impressive divergence in chain assembly, the cyclization processes and tailoring steps of the polyketide biosynthesis proposed as biosynthetic pathway for fasamycins highlights the notable control of enzymes for the aldol reaction sequence controlling the configuration of the rotationally restricted biaryl natural product.

\section{3,3'-Substituted Binaphthalenes in Catalysis}

BINAP pioneered by Noyori is established as excellent ligand for various transition metal catalyzed reactions. ${ }^{[18]}$ Nevertheless, the development of new ligands from novel scaffolds and backbone 
modifications enabled important further advances in the field of stereoselective catalysis. Wulff and co-workers recognized that the face discriminating steric shielding group in binaphthalene scaffolds is pointing away from the metal center.[19] They thus devised a new ligand bearing larger residues towards the metal center, ideally with aromatic moieties for a well-defined shielding wall. They synthesized a biphenanthrol ligand (VAPOL) which was found to be an excellent ligand for Lewis acid catalyzed Diels-Alder reactions yielding the exo-product in excellent yields, with excellent stereoselectivities (Figure 9).

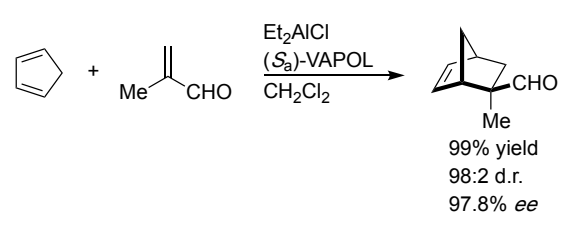

Figure 9: Lewis acid catalyzed Diels-Alder reaction with $\left(S_{a}\right)$-VAPOL as ligand.

Alternatively to the phenanthrene scaffold, the introduction of substituents in the 3,3'-position of the binaphthalene scaffold was recognized to have a profound effect on the stereoselectivity in various catalytic methods. For instance, a low enantiomeric excess of only $6 \%$ was observed in an imine reduction catalyzed by a BINOL-derived phosphoric acid, which was investigated by the List group. [20] Significantly higher selectivities were obtained with phosphoric acids bearing aryl substituents in the 3,3'position of the binaphthalene core and a major increase to an enantiomeric excess of $81 \%$ ee was obtained with $(R)$-TRIP containing triisopropyl-equipped aryl substituents (Figure 10a). A clear trend was also observed by Maruoka and co-workers by the development of new ion-pairing catalysts.[21] The already suitable enantioselectivity of $73 \%$ ee observed in the preparation of an $\alpha$-amino acid with a catalyst based on an unsubstituted binaphthalene core was further increased to an excellent enantiomeric excess of $99 \%$ through the introduction of electron deficient aryl groups in the 3,3'-positions of the binaphthalene scaffold of the catalyst (Figure 10b). Furthermore, the Cramer group reported a rhodium catalyzed $\mathrm{C}-\mathrm{H}$ activation with chiral $\mathrm{Cp}$ ligands where the stereoselective outcome was again markedly influenced by the 3,3 '-substituents. A TIPS protected binaphthol backbone provided the allylated benzamide in $80 \%$ ee compared to $35 \%$ ee obtained with an unsubstituted ligand.[22] Further optimization of the conditions allowed to prepare the desired product with up to $93 \%$ enantiomeric excess (Figure 10c). The 3,3'-substituted binaphthalene scaffold has become indispensable for phosphoric acid-,[23] ion-pairing-[24] and transition metal catalysis.[25] However, the considerable synthetic challenge to introduce substituents in the 3,3'-position of the binaphthalene core is limiting the applicability of these scaffolds. The protection of the hydroxy functions of enantiomerically enriched BINOL followed by a directed ortholithiation is still a common synthetic strategy, which is suitable for BINOL-derivates for chiral phosphoric acids (Scheme 11a).[26,27] Nevertheless, the additional homologation with carbon substituents in the 2-position is required as for Maruoka's ion-pairing catalyst and the rhodium $\mathrm{Cp}^{\mathrm{x}}$ catalysts and protecting group manipulations render their synthesis relatively laborious (Figure 11b).[21,22]
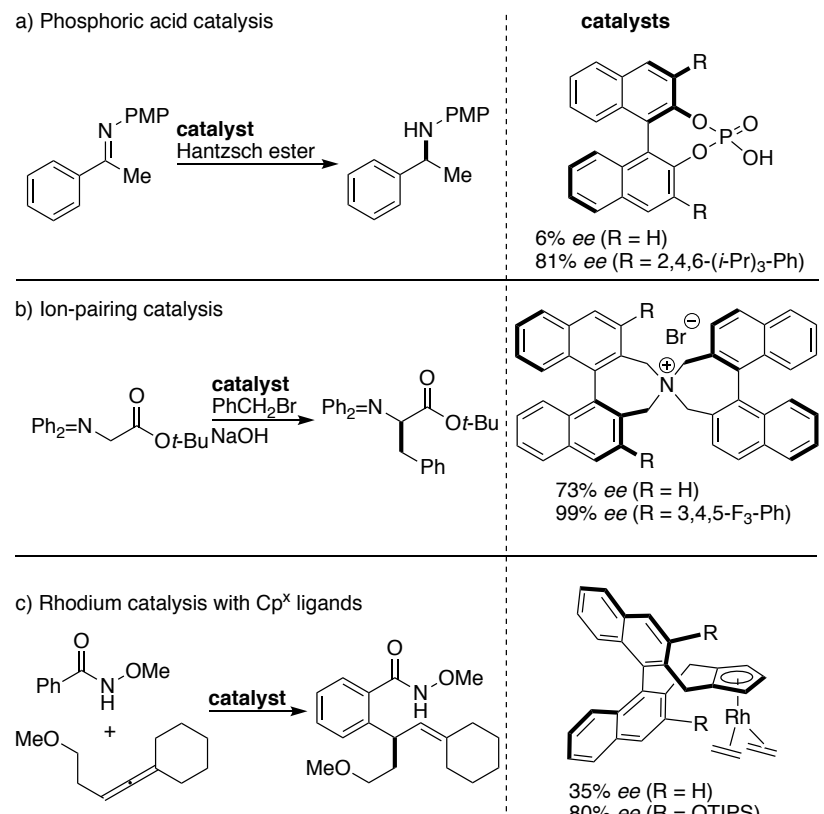

$35 \%$ ee $(\mathrm{R}=\mathrm{H})$ $80 \%$ ee $(\mathrm{R}=\mathrm{OTIPS})$

Figure 10: a) Imine reduction with chiral phosphoric acids; b) Ion-pairing catalysis for the preparation of $\alpha$-amino acid derivatives; c) Rhodium catalyzed $\mathrm{C}-\mathrm{H}$ activation for the synthesis of allylated benzamides.

a) Preparation of chiral phosphoric acids

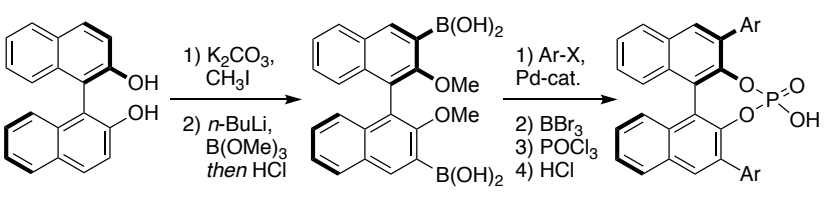

b) Preparation of Maruoka ion-pairing catalysts and rhodium $\mathrm{Cp}$ catalysts
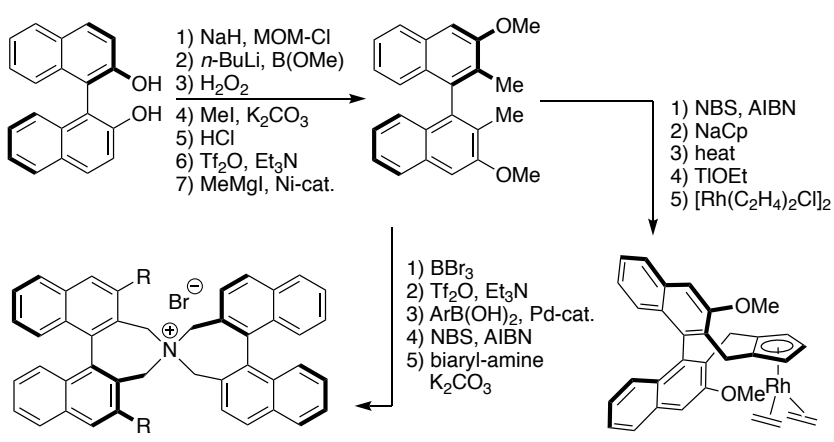

Figure 11: a) General strategy for the synthesis of 3,3'-substituted BINOL derivates to prepare chiral phosphoric acids; b) Synthesis of Maruoka ion-pairing catalysts and rhodium $\mathrm{Cp}^{\mathrm{x}}$ catalysts starting from BINOL.

More recently, Maruoka and co-workers developed an alternative strategy based on the oxidative dimerization of 2naphthoic acid to circumvent the additional steps to introduce the carbon in the 2-position of the binaphthalene. This method requires a resolution and a double directed ortho-lithiation. ${ }^{[28]} \mathrm{A}$ direct stereoselective approach to 3,3'-substituted biaryls, particularly with C-functionalized 2,2'-position therefore remains highly desirable. 


\section{Stereoselective Synthesis of Atropisomers}

The utility of atropisomers as ligand scaffolds in catalysis as well as their possible use in medicinal chemistry encouraged several groups to develop methods for the stereoselective preparation of rotationally restricted biaryls. The established catalystcontrolled methodologies typically rely on desymmetrization, kinetic resolution, the stereoselective formation of the biaryl bond, the conversion of configurationally unstable biaryls into rotationally restricted compounds and the de novo construction of arenes. ${ }^{[29-31]}$ Selected examples are outlined below with a focus on biaryl products with potential applications as ligands for stereoselective catalysis.

The substantial steric demand attributed to the orthosubstituents intrinsically lowers the reactivity towards the direct formation of the rotationally restricted axis. Thus, besides the template mediated biaryl coupling[32] and diastereoselective Ullmann reactions, ${ }^{[33]}$ enantioselective cross-coupling reactions remain challenging. ${ }^{[34]}$ Whilst the initially developed Kumada cross-coupling reactions are limited to alkyl substituents,[35] the group of Buchwald described an enantioselective cross-coupling methodology for functionalized biaryls by a Suzuki crosscoupling strategy for the synthesis of naphthyl phosphonates with high atroposelectivity. The obtained phosphonate was readily converted into a diphenyl phosphine compound as a potential mono-phosphine ligand (Figure 12).[36]
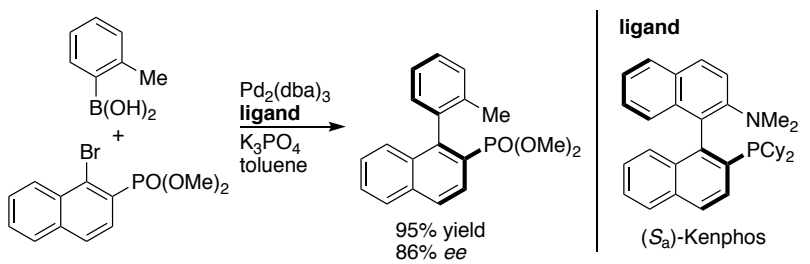

Figure 12: Atroposelective Suzuki cross-coupling reaction for the preparation of functionalized biaryls.

The preparation of tetra-ortho-substituted biaryls resulted in decreased atroposelectivities indicating the challenges for the direct formation of such stereogenic axes. Due to these difficulties, atroposelective cross-coupling reactions were complemented with an alternative method, which is inspired by oxidative dimerization of aromatic compounds as observed in the biosynthesis of numerous biaryls natural products.[37]

A strategy to circumvent the tedious formation of the sterically encumbered biaryl bond is to sufficiently substitute a configurationally labile biaryl substrate to access configurationally stable product. The evolved methodologies include the dynamic kinetic resolution (DKR) through the stereoselective introduction of ortho-substituents ${ }^{[38]}$ for instance by $\mathrm{C}-\mathrm{H}$ activation strategies. ${ }^{[39]}$ Furthermore, the interconversion of otherwise configurationally stable biaryls was realized through a planarization of the biaryl by the formation of a bridging biaryl lactone. The stereoselective lactone opening thereby allowed the atroposelective preparation of various biaryl products. ${ }^{[40]}$ A similar strategy was developed by Stoltz and Virgil for the stereoselective preparation of QUINAP. ${ }^{[41]}$ The examination of conditions revealed the possibility of a DKR starting from the racemic naphthyl triflate, which provided the
$\left(S_{\mathrm{a}}\right)$-QUINAP in $85 \%$ yield and an enantiomeric excess of $90 \%$. The formation of a square planar palladium complex with an agostic $\mathrm{C}-\mathrm{H}$ interaction was proposed to enable the rotation about the otherwise stable biaryl axis (Figure 13).

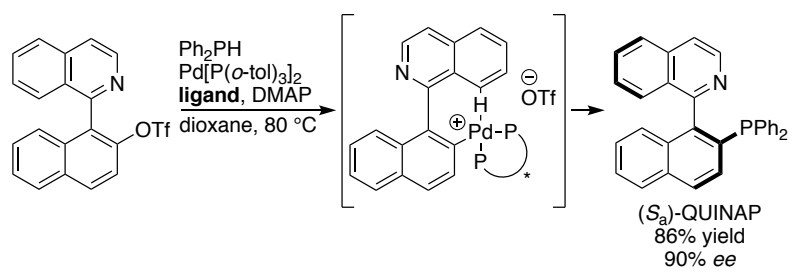

Figure 13: Palladium catalyzed DKR for the stereoselective preparation of $\left(S_{a}\right)$-QUINAP.

Controlling the configuration of the stereogenic elements by the formation of arenes emerged as a valuable strategy in the field of stereoselective catalysis.[42] To prepare molecules containing a stereogenic axis, the $[2+2+2]$-cyclotrimerization of alkyne substrates is particularly versatile concept, which has been successfully applied for the stereoselective preparation of multiaxis systems, heterocyclic biaryls and axially chiral aromatic amides.[43] Highly substituted biaryls are accessible by the $[2+2+2]$-cyclotrimerization as demonstrated by Tanaka and coworkers by the synthesis of biaryl diphosphonates as a potential precursor for a novel diphosphine ligand. The highly active rhodium catalyst allowed to scale up the reaction, which was completed within one hour yielding $18 \mathrm{~g}$ (67\% yield) of the rotationally restricted compound in an excellent enantiomeric excess of 99\% (Figure 14).

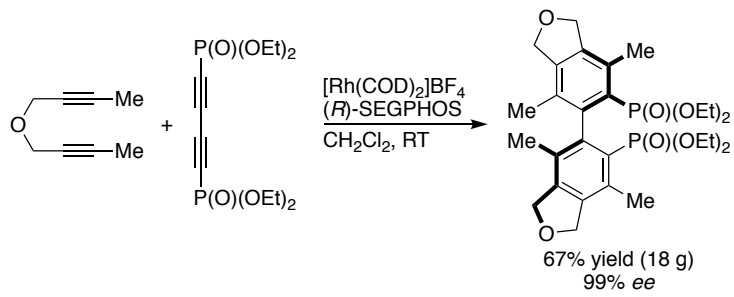

Figure 14: Rhodium catalyzed $[2+2+2]$-cyclotrimerization for the stereoselective preparation of biaryl diphosphonates.

Our group focused on the development of atroposelective areneforming aldol condensations, which were inspired by the biosynthesis of aromatic polyketides. ${ }^{[4,45]}$ The amine catalyzed aldol methodology was found to be broadly applicable permitting the preparation of binaphthalene carbaldehydes,[46] configurationally stable oligo-1,2-naphthylenes ${ }^{[47]}$, axially chiral amides $^{[48]}$ and the stereodivergent synthesis of atropisomeric multi-axis systems.[49]

\section{Synthesis of Enantioenriched Tetra-ortho-3,3'- Substituted Biaryls by the Atroposelective Arene- Forming Aldol Condensation}

These cyclization studies ${ }^{[44-49]}$ revealed the ability of smallmolecule catalysts to trigger polyketide-type cyclizations and suggested that also noncanonical polyketide cyclizations, that are related to the conversion of poly- $\beta$-carbonyl compounds by the polyketide synthase machineries, could be feasible. The synthesis 
of substrates to examine this hypothesis involved the preparation of cinnamyl biindene precursors which were accessed through an oxidative dimerization or a Suzuki crosscoupling strategy.[50] A remarkably efficient four-fold ozonolysis was observed, converting the cinnamyl biindenes into the desired noncanonical hexa-carbonyl substrates containing a 1,2diketone functionality (Figure 15).

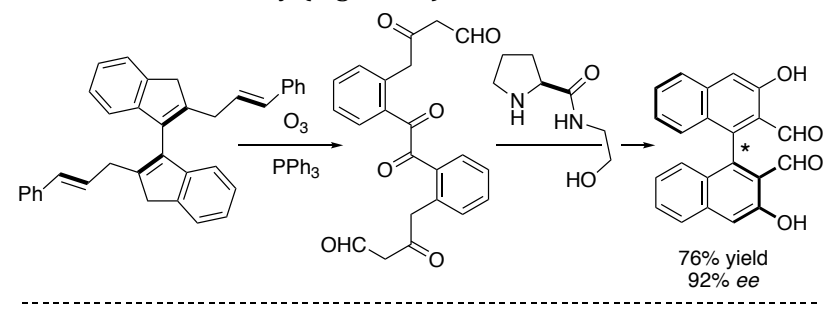

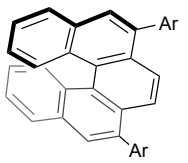

[5]helicene $96 \%$ ee

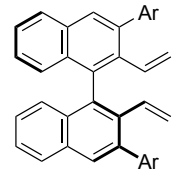

chiral diene ligand $99 \%$ ee

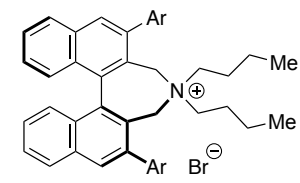

Maruoka catalyst $99 \%$ ee

Figure 15: Noncanonical polyketide cyclization for the synthesis of tetraortho-3,3'-substituted biaryls

Secondary amine catalysts bearing a hydrogen-bond donating side chain were identified to deliver the binaphthalene dicarbaldehyde products in high yields and good atroposelectivities (up to e.r. 98:2), directly resulting in the stereoselective preparation of tetra-ortho- and 3,3'-substituted biaryls. This hence allowed the synthesis of atropisomeric binaphthalene dicarbaldehydes distinct from oxidative dimerization processes and natural polyketides. Based on the mechanistic investigations of the arene-forming aldol condensation together with the observation of a symmetric reaction intermediate in NMR experiments, a twofold aldol addition followed by a double dehydration was hypothesized as a possible mechanism for the developed methodology. The utility of the developed twofold arene-forming aldol condensation could be demonstrated by the straightforward stereoselective syntheses of a [5]helicene, a chiral diene ligand and the highly enantioenriched Maruoka ion-pairing catalyst ( $99 \% e e$ ) from the 3,3'-oxygenated binaphthalene dicarbaldehyde.

\section{Conclusion}

The enantioenriched tetra-ortho- and 3,3'-substituted biaryls represent a privileged chiral scaffold ${ }^{[51]}$ for the design of catalysts used in stereoselective synthesis, as the structurally well-defined architectures provide a catalytic active site with a particularly organized shielding. However, the enantiocontrolled preparation of these valuable scaffolds remains a synthetic challenge. The oxidative dimerization of oxygenated aromatics, which is related to the tailoring processes observed in polyketide biosynthesis, provides valuable BINOL-type precursors that have served for various catalysts widely employed for stereoselective catalysis. Since several efficient approaches for the stereoselective synthesis of atropisomeric compounds have been disclosed, an enantiocontrolled and ideally direct synthesis of tetra-ortho-3,3'-

substituted biaryls is particularly advantageous. Complementing other methods, the atropisomer-selective, two-fold aldol condensation by a noncanonical polyketide cyclization provided 3,3'-oxygenated biaryls with aldehyde groups at the 2,2'positions. These orthogonal functionalities allow an efficient preparation of helicenes, ligands and valuable catalysts.

\section{Funding Information}

We gratefully acknowledge the Swiss National Science Foundation (BSSGI0-155902/1 and 175746), the University of Basel and the NCCR Molecular Systems Engineering for financial support.

\section{References}

(1) Staunton, J.; Weissman, K. J. Nat. Prod. Rep. 2001, 18, 380.

(2) Hertweck, C. Angew. Chem. Int. Ed. 2009, 48, 4688.

(3) Crawford, J. M.; Townsend, C. A. Nat. Rev. Microbiol. 2010, 8, 879.

(4) Jenke-Kodama, H.; Sandmann, A.; Müller, R.; Dittmann, E. Mol. Biol. Evol. 2005, 22, 2027.

(5) Koshla, C.; Gokhale, R. S.; Jacobsen, J. R.; Cane, D. E. Annu. Rev. Biochem. 1999, 68, 219.

(6) Austin, M. B.; Noel, J. P. Nat. Prod. Rep. 2003, 20, 79.

(7) Crawford, J. M.; Thomas, P. M.; Scheerer, J. R.; Vagstad, A. L.; Kelleher, N. L.; Townsend, C. A. Science 2008, 320, 243.

(8) Crawford, J. M.; Korman, T. P.; Labonte, J. W.; Vagstad, A. L.; Hill, E. A.; Kamari-Bidkorpeh, O.; Tsai, S.-C.; Townsend, C. A. Nature 2009, 461, 1139.

(9) Walsh, C. T.; Chen, H.; Keating, T. A.; Hubbard, B. K.; Losey, H. C.; Luo, L.; Marshall, C. G.; Miller, D. A.; Patel, H. M. Curr. Opin. Chem. Biol. 2001, 5, 525.

(10) Wang, P.; X. Gao, Y. Tang, Curr. Opin. Chem. Biol. 2012, 16, 362.

(11) Bode, S. E.; Drochner, D.; Müller, M. Angew. Chem. Int. Ed. 2007, 46, 5916.

(12) Dagne, E.; Steglich, W. Phytochemistry 1984, 23, 1729.

(13) Aldemir, H.; Richarz, R.; Gulder, T. A. M. Angew. Chem. Int. Ed. 2014, $53,8286$.

(14) Girol, C. G.; Fisch, K. M.; Heinekamp, T.; Günther, S.; Hüttel, W.; Piel, J.; Brakhage, A. A.; Müller, M. Angew. Chem. Int. Ed. 2012, 51, 9788.

(15) Mazzaferro, L. S.; Hüttel, W.; Fries, A.; Müller, M. J. Am. Chem. Soc. 2015, 137, 12289 .

(16) Qin, Z.; Munnoch, J. T.; Devine, R.; Holmes, N. A.; Seipke, R. F.; Wilkinson, K. A.; Wilkinson, B.; Hutchings, M. I. Chem. Sci. 2017, 8, 3218.

(17) Feng, Z.; Kallifidas, D.; Brady, S. F. Proc. Natl. Acad. Sci. U.S.A. 2011, 108, 12629.

(18) (a) Miyashita, A.; Yasuada, A.; Takaya, H.; Toriumi, K.; Ito, T.; Souchi, T.; Noyori, R. J. Am. Chem. Soc. 1980, 102, 7932; (b) S. Akutagawa, Appl.Catal. A, 1995, 128, 171.

(19) Bao, J.; Wulff, W. D. J. Am. Chem. Soc. 1993, 115, 3814.

(20) Hoffmann, S.; Majeed, A.; List, B. Angew. Chem. Int. Ed. 2005, 44, 7424.

(21) Ooi, T.; Kameda, M.; Maruoka, K. J. Am. Chem. Soc. 2003, 125, 5139.

(22) Ye, B.; Cramer, N. J. Am. Chem. Soc. 2013, 135, 636.

(23) Zamfir, A.; Schenker, S.; Freund, M.; Tsogoeva, S. Org. Biomol. Chem. 2010, 8, 5262-5276.

(24) Shirakawa, S.; Maruoka, K. Angew. Chem. Int. Ed. 2013, 52, 4312.

(25) Ye, B.; Cramer, N. Acc. Chem. Res. 2015, 48, 1308.

(26) Wipf, P.; Jung, J.-K. J. Org. Chem. 2000, 65, 6319.

(27) (a) Akiyama, T.; Itoh, J.; Yokota, K.; Fuchibe, K. Angew. Chem. Int. Ed. 2004, 43, 1566; (b) Various 3,3'-substituted-BINOLs and corresponding chiral phosphoric acids are now commercially available from Solvias.

(28) Kitamura, M.; Arimura, Y.; Shirakawa, S.; Maruoka, K. Tetrahedron Lett. 2008, 49, 2026.

(29) a) Bringmann, G.; Mortimer, A. J. P.; Keller, P. A.; Gresser, M. J.; Garner, J.; Breuning, M. Angew. Chem. Int. Ed. 2005, 44, 5384; b) Wencel- 
Delord, J.; Panossian, A.; Leroux, F. R.; Colobert, F. Chem. Soc. Rev. 2015, 44, 3418.

(30) Baudoin, O. Eur. J. Org. Chem. 2005, 4223.

(31) Zilate, B.; Castrogiovanni, A.; Sparr, C. ACS Catal. 2018, 8, 2981.

(32) Miyano, S.; Fukushima, H.; Handa, S.; Ito, H.; Hashimoto, H. Bull. Chem. Soc. Jpn. 1988, 61, 3249.

(33) Andrus, M. B.; Asgari, D.; Scafani, J. A. J. Org. Chem. 1997, 62, 9365.

(34) Nicolaou, K. C.; Natarajan, S.; Li, H.; Jain, N. F.; Hughes, R.; Solomon, M. E.; Ramanjulu, J. M.; Boddy, C. N. C.; Takayanagi, M. Angew. Chem. Int. Ed. 1998, 37, 2708.

(35) Hayashi, T.; Hayashizaki, K.; Kiyoi, T.; Ito, Y. J. Am. Chem. Soc. 1988, 110,8153

(36) Yin, J.; Buchwald, S. L. J. Am. Chem. Soc. 2000, 122, 12051.

(37) Kozlowski, M. C.; Morgan, B. J.; Linton, E. C. Chem. Soc. Rev. 2009, 38, 3193.

(38) Gustafson, J. L.; Lim, D.; Miller, S. J. Science 2010, 328, 1251.

(39) Yao, Q.-J.; Zhang, S.; Zhan, B.-B.; Shi, B.-F. Angew. Chem. Int. Ed. 2017, $57,6617$.
(40) Bringmann, G.; Gulder, T.; Gulder, T. A. M.; Breuning, M. Chem. Rev. 2011, 111, 563.

(41) Bhat, V.; Wang, S.; Stoltz, B. M.; Virgil, S. C. J. Am. Chem. Soc. 2013, 135, 16829.

(42) Link, A.; Sparr, C. Chem. Soc. Rev. 2018, 47, 3804.

(43) Tanaka, K. Chem. Asian J. 2009, 4, 508.

(44) Witzig, R. M.; Lotter, D.; Fäseke, V. C.; Sparr, C. Chem. Eur. J. 2017, 23, 12960.

(45) Fäseke, V. C.; Witzig, R. M.; Link, A.; Sparr, C. Chimia 2017, 71, 596.

(46) Link, A.; Sparr, C. Angew. Chem. Int. Ed. 2014, 53, 5458.

(47) Lotter, D.; Neuburger, M.; Rickhaus, M.; Häussinger, D.; Sparr, C. Angew. Chem. Int. Ed. 2016, 55, 2920.

(48) Fäseke, V. C.; Sparr, C. Angew. Chem. Int. Ed. 2016, 55, 7261.

(49) Lotter, D.; Castrogiovanni, A.; Neuburger, M.; Sparr, C. ACS Cent. Sci. 2018, 4, 656

(50) Witzig, R. M.; Fäseke, V. C.; Sparr, C. Nature Catal. 2019, 2, in print.

(51) Yoon, T. P.; Jacobsen, E. N. Science 2003, 299, 1691.

\section{Biosketches}

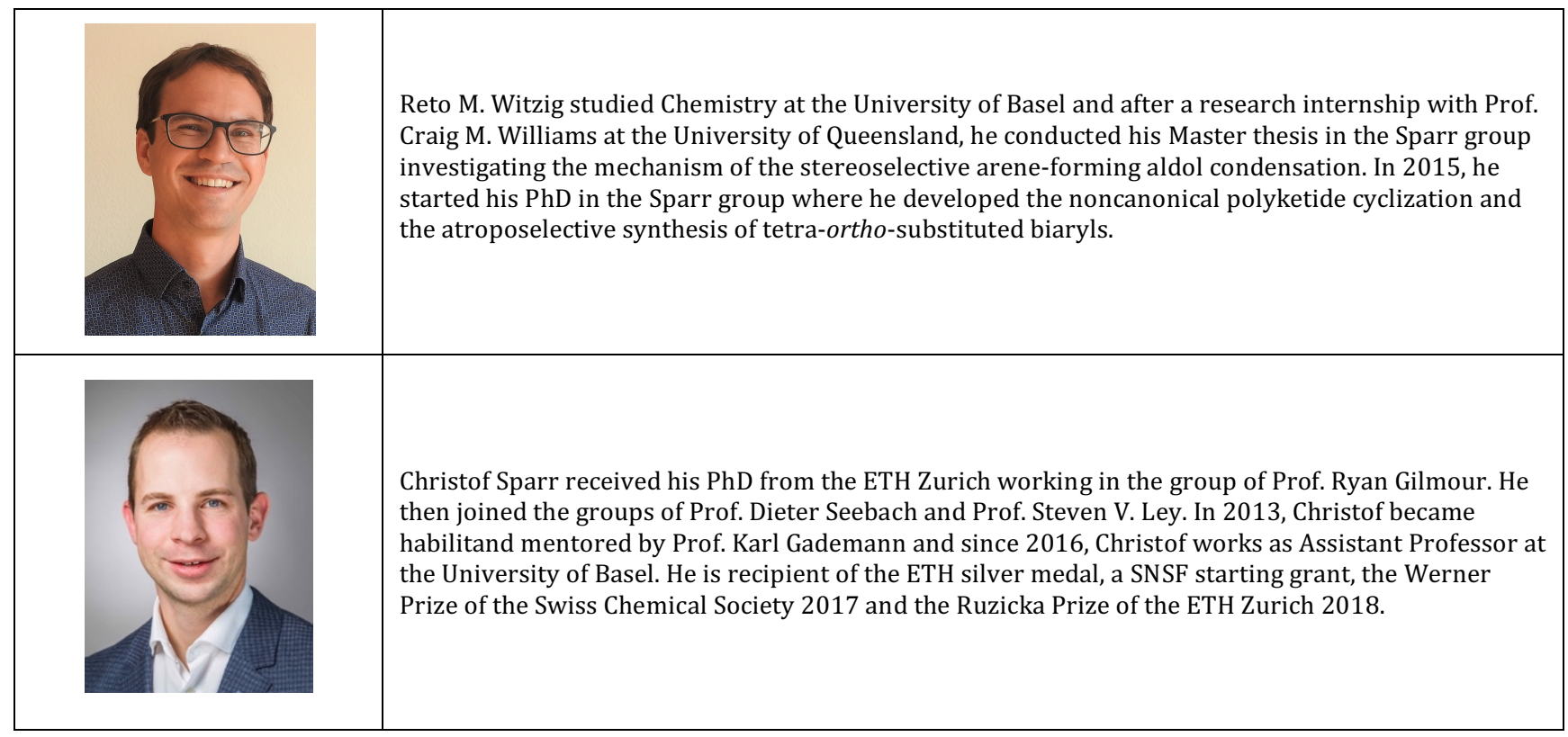

